\title{
NOVA OUTBURSTS AND THE SECULAR EVOLUTION OF CATACLYSMIC VARIABLES
}

\author{
K. SCHENKER ${ }^{1}$, U. KOLB ${ }^{1,2}$, H. RITTER ${ }^{1}$ \\ 1. MPI für Astrophysik, Garching, Germany \\ 2. Lick Observatory, UCSC, Santa Cruz, CA 95064, USA
}

\begin{abstract}
We present calculations of the long-term evolution of CVs which include the influence of nova outbursts. In particular we investigate the consequences of the discontinuous mass loss due to recurring outburst events and the effects of frictional angular momentum loss (FAML), i.e. the interaction of the expanding nova envelope with the secondary. We show that a description assuming continuous mass loss - averaged over a complete nova cycle - is applicable for determining the mean mass transfer rate and the secular evolution both with and without FAML. Between two subsequent outbursts, deviations from the mean evolution depend on the strength of FAML and on the mass ejected during the outburst. Formally FAML is a consequential angular momentum loss [1] and therefore increases the mean mass transfer rate by pushing the systems closer to mass transfer instability. Depending on the actual strenghth of FAML the long-term evolution of CVs could be significantly different from the standard model predictions.
\end{abstract}

\section{Physical situation and model calculations}

Typical model computations of the long-term evolution of CVs take into account classical nova outbursts only as a source of a continuous isotropic stellar wind from the white dwarf (WD). In contrast to that, the real physical situation is as follows. During an outburst a mass $\Delta M_{\text {ejc }}$ is removed from the system with the WD's specific angular momentum $j_{1}$. Although this event is practically instantaneous compared to the recurrence time of nova outbursts $\left(\tau_{\text {rec }} \sim 10^{3} \ldots 10^{6} \mathrm{yr}\right)$ it results in a short common envelope phase, during which angular momentum is transferred from the orbit to the envelope [5]. In the simplified model used here [4] this additional angular momentum loss depends mainly on the ratio $K_{1}=v_{\exp } / v_{\mathrm{sec}}$ of the velocity of the expanding envelope near the secondary, $v_{\exp }$, and the secondary's orbital velocity, $v_{\mathrm{sec}}$. The final specific angular momentum of the ejected matter is then $j_{\mathrm{ejc}}=j_{1}+j \mathcal{A}(q) \sqrt{1+K_{1}^{2}} / K_{1}$, where $j$ is the specific orbital 447

A. Evans and J. H. Wood (eds.), Cataclysmic Variables and Related Objects, 447-448. (C) 1996 Kluwer Academic Publishers. Printed in the Netherlands. 
angular momentum of the system and the function $\mathcal{A} \sim 0.1 \ldots 0.2$ depends only weakly on the mass ratio, $q=M_{1} / M_{2}$.

Full resolution of nova outbursts requires a time step significantly smaller than the recurrence time, whereas a complete evolution can last for more than $10^{10}$ yr. Thus complete secular evolution calculations have been performed with a fast bipolytrope code [3], short sections and averaged models also with a full stellar evolution code [6].

\section{Results and discussion}

In the fully resolved calculations all outburst parameters were taken from nova models [7] and the strength of FAML was calculated with constant values of $v_{\exp }$. These calculations yield two types of cyclic behavior, either (i) a sudden decrease in the mass transfer rate, $\dot{M}$, due to the outburst followed by a slow increase on a time-scale of $\sim 10^{4} \mathrm{yr}$ until the next outburst (no or weak FAML), or (ii) a sudden increase followed by a slow decrease (strong FAML). Both types can maintain stable mass transfer for the whole secular evolution of the system. Comparing the explicitly resolved calculations to sequences obtained with averaged continuous mass loss confirms the validity of the latter to determine the mean evolution and mass transfer rate. The amplitude of the modulation of $\dot{M}$ can be estimated from outburst parameters [8].

A realistic description should also consider how FAML varies during the secular evolution. Accordingly we have performed averaged calculations using a variable $v_{\text {exp }}$. If FAML grows sufficiently in strength with decreasing orbital separation (mainly because $v_{\exp }$ is expected to be smaller closer to the WD) mass transfer becomes adiabatically unstable, leading to a runaway where $\dot{M}$ grows orders of magnitude within a very short time until FAML disappears or a common envelope forms [2]. Furthermore a dependence of $v_{\exp }$ on outburst characteristics introduces a feedback mechanism, e.g. in the sense that a higher $\dot{M}$ causes less violent novae with slower expansion and thus stronger FAML, unless $\dot{M}$ is so high that the FAML effect disappears altogether because the nova envelope does not reach the secondary. This can lead to a very complex evolution with various detached phases.

\section{References}

1. King, A.R., Kolb, U., 1995, Ap. J., 439, 330

2. Kolb, U., Livio, M., Schenker, K., Ritter, H., 1996, in preparation

3. Kolb, U., Ritter, H., 1992, A\&A, 254, 213

4. Livio, M., Govarie, A., Ritter, H., 1991, A\&A, 246, 84

5. MacDonald, J., 1986, Ap. J., 305, 251

6. Mazzitelli, I., 1989, Ap. J., 340, 249

7. Prialnik, D., Kovetz, A., 1995, Ap. J., 445, 789

8. Schenker, K., Kolb, U., Ritter, H., 1996., in preparation 